\title{
More on SARS-COV-2 infection in children and adolescents
}

(c) The Author(s), under exclusive licence to the International Pediatric Research Foundation, Inc 2021

Pediatric Research (2022) 91:472; https://doi.org/10.1038/s41390021-01723-z

We read with great interest the study by Mantovani et al. (Vol. 89, issue 4) ${ }^{1}$. The authors aimed to assess the overall prevalence of clinical signs, symptoms and radiological findings in children and/or adolescents with COVID-19. We want to congratulate the authors for establishing a timely and informative systematic review of COVID-19 in children. Certainly, the findings of Mantovani et al. hold significance for pediatricians. However, we would like to draw their attention to the following issues.

First, according to the exclusion criteria, the authors stated that 'papers in non-English language were excluded'. However, the references 21,27 and 28 included in this study were all published in Chinese.

Furthermore, the authors failed to exclude studies suspected of including duplicate reporting. Some children or adolescents may have been included in multiple publications, as admission dates overlap for reports from the same hospital. Several studies were published by the same hospital with the periods of recruitment overlapped $^{2-4}$. Including duplicates may introduce bias into a systematic review and may affect understanding the disease and its epidemiology ${ }^{5}$. Studies suspected of including duplicate reporting can be identified based on hospital location, participant admission date, maternal and neonatal characteristics, and outcomes. Thus, we suggest that the authors extract the setting/ hospital and recruitment periods to reduce the overlapping risk ${ }^{6}$.

\footnotetext{
Jianghui Cai ${ }^{1}$, Yonghong $\operatorname{Lin}^{1}$ and Meng-jun $\mathrm{Wu}^{1 \mathrm{凶}}$ ${ }^{1}$ Chengdu Women's and Children's Central Hospital, School of Medicine, University of Electronic Science and Technology of China, Chengdu, China. ${ }^{\bowtie}$ email: wumengjuncd@126.com
}

\section{REFERENCES}

1. Mantovani, A. et al. Coronavirus disease 2019 (COVID-19) in children and/or adolescents: a meta-analysis. Pediatr. Res. 89, 733-737 (2021).

2. Zheng, F. et al. Clinical characteristics of children with coronavirus disease 2019 in Hubei. China Curr. Med. Sci. 40, 275-280 (2020).

3. Sun, D. et al. Clinical features of severe pediatric patients with coronavirus disease 2019 in Wuhan: a single center's observational study. World J. Pediatr. 16, 251-259 (2020).

4. Lu, X. et al. SARS-CoV-2 infection in child. N. Engl. J. Med. 382, 1663-1665 (2020).

5. Bauchner, H., Golub, R. M. \& Zylke, J. Editorial concern-possible reporting of the same patients with COVID-19 in different reports. JAMA 323, 1256 (2020).

6. Cai, J. et al. Cesarean section or vaginal delivery to prevent possible vertical transmission from a pregnant mother confirmed with COVID-19 to a neonate: a systematic review. Front. Med. (Lausanne) 8, 634949 (2021).

\section{AUTHOR CONTRIBUTIONS}

M.-j.W, conceived the design of the comments and made critical revision. J.C. and Y.L. drafted the manuscript. All authors approved the final version for publication.

\section{COMPETING INTERESTS}

The authors declare no competing interests.

\section{ADDITIONAL INFORMATION}

Correspondence and requests for materials should be addressed to M.-j.W.

Reprints and permission information is available at http://www.nature.com/ reprints

Publisher's note Springer Nature remains neutral with regard to jurisdictional claims in published maps and institutional affiliations. 